\title{
Diagnostic Accuracy of Ultrasonography and Magnetic Resonance Imaging in the Assessment of Placenta Previa Accreta
}

\author{
(1) Ebru ALICI DAVUTOĞLU, \\ (D) Hatice ARIÖZ HABIBi, ${ }^{b}$ \\ (D) Ayşegül ÖZEL, ${ }^{a}$ \\ (D) Hakan ERENEL, ${ }^{a}$ \\ (1) İbrahim ADALETLi ${ }^{b}$ \\ (D) Rıza MADAZLIa \\ Departments of \\ abstetrics and Gynecology, \\ ${ }^{\mathrm{b}}$ Radiology, \\ İstanbul University-Cerrahpaşa \\ Cerrahpaşa Faculty of Medicine, \\ İstanbul
}

Received: 17.06.2018

Received in revised form: 31.08 .2018

Accepted: 03.09.2018

Available online: 25.10 .2018

\section{Correspondence:}

RIza MADAZLI

İstanbul University-Cerrahpaşa

Cerrahpaşa Faculty of Medicine,

Department of Obstetrics and Gynecology,

İstanbul, TURKEY

madazli@superonline.com

Copyright $\odot 2018$ by Türkiye Klinikleri

\begin{abstract}
Objective: The present study evaluated the ability of magnetic resonance imaging (MRI) andultrasonography (US) in diagnosing placenta accreta in cases of anteriorly localized placenta previa with a high risk of placental adhesion abnormality. Material and Methods: A total of 29 patients with anteriorly localized placenta previa and having at least one risk factor for placenta accreta underwent US and MRI. Diagnostic ability of both modalities for the prediction of abnormal placental invasiveness was assessed using various imaging signs described in the existing literature. Results: Of the 29 pregnancies with placenta previa, 13 (44.8\%) had placenta accreta that was confirmed at the time of surgery. Cesarean hysterectomy was performed in seven (53.8\%) cases. Sensitivity, specificity, and diagnostic accuracy of ultrasound and MRI were calculated to be $84.6 \%$, $81.2 \%$, and $82.7 \%$ and $100 \%, 76.9 \%$, and $86.2 \%$, respectively. In 5 of 29 cases, US and MRI had discordant diagnoses; sonography detected placenta accreta in 2 cases, whereas magnetic resonance imaging predicted it in 3 cases. Conclusion: The diagnostic abilities of ultrasound and MRI for the detection of placenta accreta appear to be comparable. Magnetic resonance imaging may be required to plan the surgical approach and assess the risk of potential surgical morbidity.
\end{abstract}

Keywords: Placenta accreta; placenta previa; ultrasonography; magnetic resonance imaging

$\mathrm{T}$ he term "placenta accreta" refers to a spectrum of conditions including accreta, increta, and percreta, as well as cases of the clinically apparent morbidly adherent placenta. ${ }^{1}$ Placenta previa is more commonly associated with placenta accreta as the lower segment is an area of relatively poorer decidualization and is associated with a thin or absent decidua basalis. ${ }^{2}$ Placenta previa accreta is associated with significant maternal morbidity and mortality owing to bladder and rectum invasion, massive postpartum hemorrhage and its resultant coagulopathy, cesarean hysterectomy, and death. ${ }^{3}$ The risk of placenta accreta with placenta previa ranges from 3.3 to $67 \%$; the risk increases with the number of previous cesarean deliveries. ${ }^{4,5}$ Placenta previs accreta has become an obstetric complication that an average obstetrician is likely to encounter several times. There has been a substantial increase in its occurrence over the past 50 years and it can no longer be considered as a rare obstetric pathology. ${ }^{6}$

The antenatal diagnosis of placenta previa accreta by ultrasonography (US) or magnetic resonance imaging (MRI) techniques allows effective management planning to minimize mortality and morbidity. Ultrasonography aided by color Doppler is considered the first-line modality in the evaluation 
of invasive placenta owing to its high sensitivity and specificity rates. ${ }^{7}$ US can easily detect loss of the normal hypoechoic retroplacental myometrium zone, thinning or disruption of the hyperechoic uterine serosa-bladder interface, the presence of focal exophytic mass lesions, and the presence of lacunae in the placenta. ${ }^{8}$ Although ultrasound is the mainstay in imaging placenta accreta, MRI has been used as an adjunct in diagnosis when the ultrasound results are equivocal and/or clinical suspicion is high. Specific diagnostic features of placenta accreta on MRI imagings, such as placental heterogeneity, dark intra-placental bands, intra-placental hemorrhages, indistinct bladder wall margin, tenting of bladder or bulging contour of the uterus, are reported in the literature. ${ }^{9,10}$ Potential benefits of MRI include greater soft tissue contrast and a larger field of view as compared with sonography. Pelvic ultrasound is highly reliable in diagnosing or excluding the presence of placental adhesive disorders, whereas MRI is an excellent tool for staging and topographic evaluation of adhesive disorders. ${ }^{8}$

The present study assessed the performance of US and MRI in diagnosing placenta accreta in cases of anteriorly localized placenta previa with a high risk of placental adhesion abnormality. Specific imaging markers were evaluated to determine if certain features were more specific for the presence or absence of placenta accreta and also predicting the requirement of hysterectomy in patients.

\section{MATERIAL AND METHODS}

The current study was a prospective observational case control study performed between January 2015 and November 2016 at the Cerrahpasa Medical Faculty Departments of Obstetrics and Gynecology and Radiology. The study was approved by the institutional ethics committee and was conducted in accordance with the principles of the Declaration of Helsinki, 2008. Written informed consent was obtained from all patients. A total of 29 patients with anteriorly localized placenta previa and having at least one risk factor for placenta accreta, such as the history of cesarean delivery, curettage or myomectomy, were recruited. Exclu- sion criteria included taking medication, smoking, high blood pressure, preeclampsia, gestational diabetes mellitus, multiple gestations, contraindication for MRI (presence of metallic implants, claustrophobia), and fetal chromosomal or structural anomalies. All women were followed up at the perinatology unit, and the timing of delivery was individualized for each patient. The diagnosis of placenta accreta was confirmed at the time of surgery and then again on pathology if a pathological specimen was available. All cesarean surgeries were performed by experienced obstetricians. Based on the intraoperative findings, the decision of conservative surgery was taken by the surgical team at the time of operation.

Ultrasonographic examinations were performed between 24 and 34 weeks of gestation (mean: $29.4 \pm 2.9$ weeks), and MRI examinations were performed between 28 and 36 weeks of gestation (mean: $30.4 \pm 2.4$ weeks). Ultrasonographic evaluation of the pregnancies was performed using Xario (Toshiba Medical systems; Chiba, Japan) by two experienced perinatologists (E.D. and R.M.) at our obstetric department by both grayscale and color Doppler sonography. MRI examinations of the placentas were performed at the radiology department by two experienced radiologists (H.A. and I.A.) on a 1.5-T MRI machine (Magnetom Avento; Siemens Medical System, Erlangen, Germany) with body array coils. The protocol included routine axial, sagittal T2 HASTE, coronal T2 HASTE fat sat, axial T1 turbo spin echo, and axial T1 VIBE fat sat. Images were obtained with 6-mm slice thickness. Also, diffusion-weighted images with ADC map, b50, b400, and b800 were obtained. No contrast medium was administered during MRI examination. Ultrasonographic examination included standard fetal biometry, anomaly scan, and placental localization and morphology. Placenta previa was diagnosed after 22 weeks of gestation and confirmed by transvaginal ultrasonography. Findings suggestive of placenta accreta included loss of myometrial interface or retroplacental clear space, reduced myometrial thickness less than $1 \mathrm{~mm}$, loss of the bladder walluterine border, presence of a focal exophytic mass 
with the same echogenicity as placenta beyond the uterine serosa, placental lacunae, chaotic intra-placental blood flow and intra-placental lacunae, bladder uterine serosa interface hypervascularity, vessels extending from placenta to bladder, and vessels bridging from placenta to margin of uterus. ${ }^{8,9}$ The cases were marked as negative for invasive placenta only when all signs were absent on sonography. All cases with placenta previa were reevaluated by MRI during the third trimester. MRI features that were identified as invasive placenta included placental heterogeneity, dark intra-placental bands, intra-placental hemorrhages, focal interruption of the myometrium and tenting of the bladder, and uterine bulging. ${ }^{89}$ Again, the cases were marked negative for placenta accreta only when all the described set of criteria was absent in the patient. The US and MRI findings were compared with clinical and pathologic outcomes and classified as true positive (TP), true negative (TN), false positive (FP), and false negative (FN). Uncomplicated placental removal without excessive bleeding after cesarean delivery was designated as TN. Patients showing a difficult placental separation with excessive bleeding from placenta bed during cesarean surgery and additional procedures (conservatively or hysterectomy) were classified as TP.

\section{STATISTICAL ANALYSIS}

All analyses were performed using the Statistical Package for the Social Science (SPSS) software version 21 (Chicago, Illinois, United States). The Kolmogorov-Smirnov test was used to assess the normality of the distribution of variables. Data were presented as a mean \pm standard deviation. Mean values between the groups were analyzed by Student's $t$-test. The chi-square test was used for comparisons of categorical variables; data were presented as proportions. The sensitivity, specificity, positive predictive value, negative predictive value, and diagnostic accuracy of US and MRI in the identification of placental abnormalities were calculated.

\section{RESULTS}

Table 1 depicts clinical characteristics and obstetric outcomes of pregnancies with placenta previa with and without accreta. Of the 29 pregnancies with placenta previa, $13(44.8 \%)$ had placenta accreta that was confirmed at the time of surgery. Pregnancies with and without placenta accreta were comparable with regard to maternal age and Apgar scores at the 5 th $\min (p>0.05)$. The mean gestational age at delivery and birth weight were significantly lower in the placenta previa with accreta group than in the group without accreta $(p<0.05)$.

TABLE 1: Clinical characteristics and obstetric outcomes of women with placenta previa with and without placenta accreta.

\begin{tabular}{|c|c|c|c|}
\hline & With accreta & Without accreta & $p$ \\
\hline Subjects (n) & 13 & 16 & \\
\hline Maternal age (years, mean $\pm S D$ ) & $31.7 \pm 4.2$ & $30.9 \pm 5.9$ & 0.701 \\
\hline Parity (mean $\pm S D$ ) & $2.2 \pm 0.7$ & $1.1 \pm 0.7$ & 0.003 \\
\hline \multicolumn{4}{|l|}{ Prior cesarean delivery $(n, \%)$} \\
\hline 1 & $13,(100 \%)$ & $11,(68.7 \%)$ & 0.029 \\
\hline$\geq 2$ & $11,(84.6 \%)$ & $3,(18.6 \%)$ & 0.001 \\
\hline Gestational age at delivery (weeks, mean $\pm S D$ ) & $34.6 \pm 5.0$ & $37.7 \pm 0.8$ & 0.023 \\
\hline Birth weight $(g$, mean $\pm S D)$ & $2.458 \pm 1.123$ & $3.235 \pm 1.123$ & 0.001 \\
\hline Apgar at 5 min (mean $\pm S D)$ & $9.0 \pm 0.6$ & $8.7 \pm 0.7$ & 0.429 \\
\hline Blood transfusions $(n, \%)$ & $9,(69.2 \%)$ & $1,(7.7 \%)$ & 0.007 \\
\hline Cesarean hysterectomy $(n, \%)$ & $7,(53.8 \%)$ & - & \\
\hline Bladder injury $(n, \%)$ & $3,(23.1 \%)$ & - & \\
\hline Perinatal mortality $(n, \%)$ & $2,(15.4 \%)$ & - & \\
\hline
\end{tabular}


Incidences of prior cesarean delivery and maternal blood transfusions were significantly higher in the placenta accreta group $(p<0.01)$. Cesarean hysterectomy was performed in seven (53.8\%) of the placenta accreta cases; the rest were managed by a conservative approach such as conservative surgery, prophylactic uterine arteries ligation, external (B-Lynch suture), and internal (Bakri balloon) uterine compression. Bladder injury occurred in three $(23.1 \%)$ patients with accreta, and primary repair was performed for all cases. There was no maternal mortality. There were two perinatal mortalities due to prematurity (23 and 31 weeks) in the placenta accreta group.

Table 2 presents the details of ultrasonographic and MRI features of the cases. Out of 13 patients

\begin{tabular}{|c|c|c|c|c|c|}
\hline Case \# & US features & MRI features & Surgery & Diagnostic accuracy USG & MRI \\
\hline \multicolumn{6}{|c|}{ Cases without accreta } \\
\hline 1 & - & - & $\mathrm{C} / \mathrm{S}$ & TN & TN \\
\hline 3 & - & - & $\mathrm{C} / \mathrm{S}$ & TN & TN \\
\hline 4 & - & - & $\mathrm{C} / \mathrm{S}$ & TN & TN \\
\hline 6 & - & - & $\mathrm{C} / \mathrm{S}$ & TN & TN \\
\hline 8 & - & - & $\mathrm{C} / \mathrm{S}$ & TN & $\mathrm{TN}$ \\
\hline 10 & - & - & $\mathrm{C} / \mathrm{S}$ & TN & TN \\
\hline 13 & - & - & $\mathrm{C} / \mathrm{S}$ & TN & $\mathrm{TN}$ \\
\hline 15 & - & $e, f$ & $\mathrm{C} / \mathrm{S}$ & TN & FP \\
\hline 17 & $a, c$ & $e, f$ & $\mathrm{C} / \mathrm{S}$ & FP & FP \\
\hline 18 & a & $e, f$ & $\mathrm{C} / \mathrm{S}$ & FP & FP \\
\hline 22 & - & - & $\mathrm{C} / \mathrm{S}$ & TN & $\mathrm{TN}$ \\
\hline 23 & - & - & $\mathrm{C} / \mathrm{S}$ & TN & TN \\
\hline 24 & - & - & $\mathrm{C} / \mathrm{S}$ & TN & $\mathrm{TN}$ \\
\hline 27 & - & - & $\mathrm{C} / \mathrm{S}$ & TN & TN \\
\hline 28 & $a, c$ & - & $\mathrm{C} / \mathrm{S}$ & FP & $\mathrm{TN}$ \\
\hline 29 & - & $e, f, g$ & $\mathrm{C} / \mathrm{S}$ & TN & FP \\
\hline \multicolumn{6}{|l|}{15} \\
\hline \multicolumn{6}{|c|}{ Cases with acreata } \\
\hline 2 & $a, b, c, d$ & $e, f, h, i$ & C/S -hyst & TP & TP \\
\hline 5 & $a, c$ & $e, f$ & C/S-CO & TP & TP \\
\hline 7 & $a, b, c, d$ & $e, f, h, i$ & C/S -hyst & TP & TP \\
\hline 9 & - & $\mathrm{f}, \mathrm{g}$ & $\mathrm{C} / \mathrm{S}+\mathrm{CO}$ & FN & TP \\
\hline 11 & - & $e, f$ & $\mathrm{C} / \mathrm{S}+\mathrm{CO}$ & FN & TP \\
\hline 12 & $a, c$ & $e, f, g$ & $\mathrm{C} / \mathrm{S}+\mathrm{CO}$ & TP & TP \\
\hline 14 & $a, b, d$ & $e, f, h, i$ & C/S-hyst & TP & TP \\
\hline 16 & $a, b, c, d$ & $e, f, h, i$ & C/S-hyst & TP & TP \\
\hline 19 & $a, b, c, d$ & $e, f, h, i$ & C/S-hyst & TP & TP \\
\hline 20 & $a, b, d$ & $e, f, h, i$ & C/S -hyst & TP & TP \\
\hline 21 & $a, b, d$ & $e, f, h, i$ & C/S -hyst & TP & TP \\
\hline 25 & $a, c$ & $e, f, g$ & $\mathrm{C} / \mathrm{S}+\mathrm{CO}$ & TP & TP \\
\hline 26 & $a, c$ & $e, f$ & $\mathrm{C} / \mathrm{S}+\mathrm{CO}$ & TP & TP \\
\hline
\end{tabular}

USG features: a: loss of myometrial interface or retroplacental clear space, reduced myometrial thickness < $1 \mathrm{~mm}$, loss of the bladder wall-uterine border; $\mathbf{b}$ : presence of a focal exophytic mass with the same echogenicity as placenta beyond the uterine serosa; $\mathbf{c}$ : placental lacunae, chaotic intraplacental blood flow and intra-placental lacunae; $\mathbf{d}$ : bladder uterine serosa interface hypervascularity, vessels extending from placenta to bladder, MRI features; e:placental heterogeneity; f: dark intraplacental bands; g: intraplacental hemorrhages; $\mathbf{h}$ : focal interruption of the myometrium and tenting of the bladder; i: bulging uterine contour; C/S-hyst: Cesarean hysterectomy; C/S+CO: Cesarean+ conservative options; TP: true positive;TN: true negative; FP: false positive; FN: false negative. 
with placenta accreta, 11 were predicted correctly based on sonography (true positive), whereas two were misdiagnosed as normal placenta (false negative). The sensitivity, specificity, and diagnostic accuracy of sonography in the identification of placenta accreta were $84.6 \%, 81.2 \%$ and $82.7 \%$, respectively (Table 3). MRI predicted four false-positive results and no false-negative results. The sensitivity, specificity, and diagnostic accuracy of MRI in the identification of placenta accreta were $100 \%, 76.9 \%$ and $86.2 \%$, respectively (Table 3 ). The invasiveness was diagnosed incorrectly by both US and MRI in two cases (false positive). In 5 of 29 cases, sonography and MRI had discordant diagnoses; sonography correctly predicted in two cases, whereas MRI correctly diagnosed in three cases.

\begin{tabular}{|lcc|}
\hline \multicolumn{3}{|c|}{$\begin{array}{c}\text { TABLO 3: Sensitivities, specificities and predictive values of ul- } \\
\text { trasound (US) and magnetic resonance imaging (MRI) } \\
\text { in the diagnosis of placenta accreta. }\end{array}$} \\
\hline \multicolumn{3}{|c|}{ US } \\
True positive & 11 & MRI \\
False positive & 3 & 13 \\
True negative & 13 & 4 \\
False negative & 2 & 12 \\
Sensitivity (\%) & 84,6 & - \\
Specificity (\%) & 81.2 & 700 \\
PPV (\%) & 78.5 & 76.4 \\
NPV (\%) & 86.6 & 100 \\
Diagnostic accuracy (\%) & 82.7 & 86.2 \\
\hline
\end{tabular}

Table 4 demonstrates the incidence of ultrasonographic and MRI features in pregnancies with placenta previa with and without placenta accreta. The loss of myometrial interface or retroplacental clear space reduced the myometrial thickness to less than $1 \mathrm{~mm}$, and the loss of the bladder walluterine border was the most frequent feature detected by US in our series ( $n=14 ; 11$ true positives and 3 false positives). A lacune was the second frequent feature detected by US ( $n=10 ; 8$ true positives and 2 false positives). Placental heterogeneity and dark intra-placental bands were the most frequent features detected by MRI ( $n=17 ; 13$ true positives and 4 false positives). Among the individual sonographic features mentioned for the detection of the invasive placenta, focal exophytic mass with the same echogenicity as placenta beyond the uterine serosa and bladder uterine serosa interface hypervascularity, and vessels extending from the placenta to bladder were the most accurate signs for detecting patients undergoing a hysterectomy. Focal interruption of the myometrium, tenting of the bladder, and bulging uterine contour were detected by MRI in all cases requiring a hysterectomy.

\section{DISCUSSION}

Placenta previa accreta is a severe pregnancy condition in which the placenta attaches itself too deeply into the uterine wall. The present study evaluated the diagnostic ability of US and MRI to

TABLO 4: Incidences of ultrasonographic and magnetic resonance imaging features in pregnancies with placenta previa with and without placenta accreta.

\begin{tabular}{|c|c|c|}
\hline & Placenta accreta $\mathrm{n} / \mathrm{N}, \%$ & No placenta accreta $\mathrm{n} / \mathrm{N}, \%$ \\
\hline $\begin{array}{l}\text { Ultrasound features Loss of myometrial interface or retroplacenta I clear space, reduced } \\
\text { myometrial thickness }<1 \mathrm{~mm} \text {, loss of the bladder wall-uterine border. }\end{array}$ & $11 / 13,(84.6)$ & $3 / 16,(18.8)$ \\
\hline Presence of a focal exophytic mass with the same echogenicity as placenta beyond the uterine serosa. & $7 / 13,(53.8)$ & 0 \\
\hline Placental lacunae, chaotic intraplacental blood flow and intra-placental lacunae & $8 / 13,(61.5)$ & 2/16, (12.5) \\
\hline Bladder uterine serosa interface hypervascularity, vessels extending from placenta to bladder, & $7 / 13,(53.8)$ & 0 \\
\hline MRI features Placental heterogeneity and dark intraplacental bands & $13 / 13,(100)$ & $4 / 16,(25)$ \\
\hline Intraplacental hemorrhages & $3 / 13,(23.1)$ & $1 / 16,(6.3)$ \\
\hline Focal interruption of the myometrium and tenting of the bladder & $7 / 13,(53.8)$ & 0 \\
\hline Bulging uterine contour & $7 / 13,(53.8)$ & 0 \\
\hline
\end{tabular}


predict placenta accreta. It was observed that the mean gestational age at delivery and birth weight were significantly lower and incidences of prior cesarean delivery and maternal blood transfusions were significantly higher in the placenta previa with accreta than in the group without accreta. These results are consistent with previous studies that demonstrated pregnancies complicated with placenta previa accreta to be prone to adverse outcomes, such as preterm delivery and blood loss.,3 Prior cesarean section and placenta previa are known risk factors for placenta accreta. ${ }^{6}$ In our study, among the study population with anteriorly localized placenta previa, $44.8 \%$ had morbidly adherent placenta of which $84.6 \%$ had $\geq 2$ prior cesarean deliveries. The main reason for this high incidence is that it is a selective group referred to our unit with a suspicion of invasive placentation.

Antenatal diagnosis of placental invasion using imaging techniques has the potential to improve maternal and fetal outcomes. ${ }^{11,12}$ Studies have shown that the multi-disciplinary planning and care team approach decrease the morbidity and mortality rates in pregnancies complicated by placenta previa accreta. ${ }^{2} \mathrm{We}$ observed no maternal mortality and serious maternal morbidity, mainly due to the morbidly adherent placenta before delivery. Planned cesarean hysterectomy was performed in $53.8 \%$ of our placenta accrete cases without trying to detach the placenta. Prenatal prediction of the adherent placenta with planned cesarean hysterectomy has been shown to decrease the morbidity and mortality related to the placenta accreta. $^{8}$

Prenatal sonography with grayscale and color Doppler imaging and MRI are useful methods for the diagnosis of placenta accreta. Many studies have demonstrated the ability of US and MRI in the prenatal detection of placenta accreta; however, the accuracy of US compared with MRI remains in question. A meta-analysis of 23 studies exploring the ability of sonography (grayscale and Doppler) for the prenatal prediction of placenta accreta found a sensitivity of $90.7 \%$ (95\% CI: 87.2-93.6) and specificity of $96.9 \%$ (95\% CI: $96.3-97.5) .{ }^{13} \mathrm{In}$ our group, the sensitivity and specificity of sonog- raphy in identifying placenta accreta were $84.6 \%$ and $81.2 \%$, respectively. In the present study, US reported the false-negative outcome in two patients in whom there was no deep placental invasion at the time of delivery and were treated conservatively.

Although ultrasound is considered to be a highly reliable tool for diagnosing disorders of invasive placentation, it is not entirely clear whether MRI improves the diagnostic accuracy of ultrasound. Further, there is uncertainty on which MRI signs to be used to diagnose this condition. A metaanalysis of 18 studies exploring the sensitivity and specificity of MRI for the prediction of placenta accreta reported these to be $94.4 \%$ (95\% CI: 86.0 97.9) and $84.0 \%$ (95\% CI: 76.0-89.8), respectively. ${ }^{14}$ In our group, the sensitivity and specificity of MRI in the identification of placenta accreta were $100 \%$ and $75.0 \%$, respectively. The MRI reported no false-negative and four false-positive results. Comparing US and MR findings in our series, 5 of 29 cases had discordant diagnoses, in which sonography was correct in two and MRI was correct in three cases. On the basis of our data and those from prior studies, it is difficult to determine the superiority of either technique for accurately diagnosing placenta accreta. ${ }^{15}$

Determining the depth of villous invasiveness before delivery is pivotal in planning individual management of placenta accreta. The significance of various ultrasound and MRI signs for the prenatal diagnosis of accreta placentation and assessment of the depth of villous invasiveness is not yet clear. The loss of myometrial interface or retroplacental clear space reduced the myometrial thickness to less than $1 \mathrm{~mm}$, and the loss of the bladder walluterine border was some of the most frequent features detected by US in our series. However, these features are subjective and do not clearly define the depth of invasion. Among the individual sonographic features, focal exophytic mass with the same echogenicity as placenta beyond the uterine serosa and bladder-uterine serosa interface hypervascularity, and vessels extending from the placenta to bladder were the most accurate signs for detecting deep invasion and requiring hys- 
terectomy in our group. A recent systematic review evaluated the importance of various ultrasound signs for the assessment of the depth of villous invasiveness. The study demonstrated that no ultrasound sign or a combination of ultrasound signs were specific to the depth of accreta placentation. ${ }^{1}$ Placental heterogeneity and dark intra-placental bands were the most frequent features detected by MRI in our placenta accreta cases. Derman et al. ${ }^{16}$ also demonstrated that abnormal placental vascularity and intra-placental bands are the most sensitive MRI criteria for the diagnosis of invasive placentation. However, focal interruption of the myometrium and bulging uterine contour were detected by MRI in all cases requiring a hysterectomy and were found to be the most useful signs for describing deep villous invasion in our series.

\section{CONCLUSION}

The diagnostic abilities of US and MRI for the detection of placenta accreta appear to be comparable. Sonography is easy to perform, easily available in most of the clinics, cost-effective, and readily accepted by the pregnant woman. MRI has been reported to provide an accurate anatomical description of the area invaded by the placenta. This evaluation may be required to plan the surgical ap- proach and assess the risk of potential surgical morbidity. MRI should, therefore, be considered when hysterectomy or one-step conservative surgery is indicated.

\section{Source of Finance}

During this study, no financial or spiritual support was received neither from any pharmaceutical company that has a direct connection with the research subject, nor from a company that provides or produces medical instruments and materials which may negatively affect the evaluation process of this study.

\section{Conflict of Interest}

No conflicts of interest between the authors and / or family members of the scientific and medical committee members or members of the potential conflicts of interest, counseling, expertise, working conditions, share holding and similar situations in any firm.

\section{Authorship Contributions}

Idea/Concept: Ebru Alıcı Davutoğlu, Hatice Arıöz Habibi; Design: Ebru Alıcı Davutoğlu, Hatice Arıöz Habibi, Ayșegül Özel; Control/Supervisıon: İbrahim Adaletli, Riza Madazli; Data Collection And/or Processing: Ebru Alıcı Davutoğlu, Hatice Arıöz Habibi, Ayşegül Özel; Analysis and/or Interpretation: Ebru Alıcı Davutoğlu, Hatice Arı̈̈z Habibi; Literature Review: Ebru Alıcı Davutoğlu, Hakan Erenel; Writing the Article: Ebru Alıcı Davutoğlu, Hatice Ariöz Habibi, Ayșegül Özel; Critical Review: Rıza Madazlı, İbrahim Adaletli; References and Fundings: Ayşegül Özel, Hakan Erenel; Materials: Ebru Alıcı Davutoğlu, Hatice Arı̈̈z Habibi.

\section{REFERENCES}

1. Jauniaux E, Collins SL, Jurkovic D, Burton GJ. Accreta placentation: a systematic review of prenatal ultrasound imaging and grading of villous invasiveness. Am J Obstet Gynecol 2016;215(6):712-21.

2. D'Antonio F, Palacios-Jaraquemada J, Lim PS, Forlani F, Lanzone A, Timor-Tritsch I, et al. Counseling in fetal medicine: evidence-based answers to clinical questions on morbidly adherent placenta. Ultrasound Obstet Gynecol 2016;47(3):290-301

3. Silver RM. Abnormal placentation: placenta previa, vasa previa, and placenta accreta. Obstet Gynecol 2015;126(3):654-68.

4. Belfort MA; Publications Committee, Society for Maternal-Fetal Medicine. Placenta accreta. Am J Obstet Gynecol 2010;203(5):430-9.

5. Solheim KN, Esakoff TF, Little SE, Cheng YW, Sparks $\mathrm{TN}$, Caughey AB. The effect of cesarean delivery rates on the future incidence of placenta previa, placenta accreta, and maternal mortality. J Matern Fetal Neonatal Med 2011;24(11):1341-6.
6. Jauniaux E, Jurkovic D. Placenta accreta: pathogenesis of a 20th century iatrogenic uterine disease. Placenta 2012;33(4):244-51.

7. Committee on Obstetric Practice. Committee opinion no. 529: placenta accreta. Obstet Gynecol 2012; 120(1):207-11.

8. Comstock $\mathrm{CH}$, Bronsteen RA. The antenatal diagnosis of placenta accreta. BJOG 2014;121(2):171-81.

9. Baughman WC, Corteville JE, Shah RR. Placenta accreta: spectrum of US and MR imaging findings. Radiographics 2008;28(7):1905-16.

10. Valentini AL, Gui B, Ninivaggi V, Miccò M, Giuliani M, Russo L, et al. The morbidly adherent placenta: when and what association of signs can improve MRI diagnosis? Our experience. Diagn Interv Radiol 2017;23(3):180-6.

11. Warshak CR, Ramos GA, Eskander R, Benirschke $K$, Saenz CC, Kelly TF, et al. Effect of predelivery diagnosis in 99 consecutive cases of placenta accreta. Obstet Gynecol 2010;115(1):65-9.
12. Tikkanen M, Paavonen J, Loukovaara M, Stefanovic V. Antenatal diagnosis of placenta accreta leads to reduced blood loss. Acta Obstet Gynecol Scand 2011;90(10):1140-6.

13. D'Antonio F, Jacovella C, Bhide A. Prenatal identification of invasive placentation using ultrasound: systematic review and meta-analysis. Ultrasound Obstet Gynecol 2013;42(5):509-17.

14. D'Antonio F, lacovella $\mathrm{C}$, Palacios-Jaraquemada J, Bruno $\mathrm{CH}$, Manzoli L, Bhide A. Prenatal identification of invasive placentation using magnetic resonance imaging: systematic review and meta-analysis. Ultrasound Obstet Gynecol 2014;44(1):8-16.

15. Kumar I, Verma A, Ojha R, Shukla RC, Jain M, Srivastava A. Invasive placental disorders: a prospective US and MRI comparative analysis. Acta Radiol 2016;58(1):121-8.

16. Derman AY, Nikac V, Haberman S, Zelenko N, Opsha $\mathrm{O}$, Flyer M. MRI of placenta accreta: a new imaging perspective. AJR Am J Roentgenol 2011;197(6):1514-21. 